Discrete Comput Geom 32:37-53 (2004)

DOI: $10.1007 / \mathrm{s} 00454-004-2864-\mathrm{x}$

Geometry

\title{
Computing the Writhing Number of a Polygonal Knot*
}

\author{
Pankaj K. Agarwal, ${ }^{1}$ Herbert Edelsbrunner, ${ }^{1,2}$ and Yusu Wang ${ }^{1}$ \\ ${ }^{1}$ Department of Computer Science, Duke University, \\ Durham, NC 27708, USA \\ \{pankaj,edels,wys\}@cs.duke.edu \\ ${ }^{2}$ Raindrop Geomagic, \\ Research Triangle Park, NC 27709, USA
}

\begin{abstract}
The writhing number measures the global geometry of a closed space curve or knot. We show that this measure is related to the average winding number of its Gauss map. Using this relationship, we give an algorithm for computing the writhing number for a polygonal knot with $n$ edges in time roughly proportional to $n^{1.6}$. We also implement a different, simple algorithm and provide experimental evidence for its practical efficiency.
\end{abstract}

\section{Introduction}

The writhing number is an attempt to capture the physical phenomenon that a cord tends to form loops and coils when it is twisted. We model the cord by a knot, which we define to be an oriented closed curve in three-dimensional space. We consider its two-dimensional family of parallel projections. In each projection, we count +1 or -1 for each crossing, depending on whether the overpass requires a counterclockwise or a clockwise rotation (an an angle between 0 and $\pi$ ) to align with the underpass. The writhing number is then the signed number of crossings averaged over all parallel projections. It is a conformal invariant of the knot and useful as a measure of its global geometry.

The writhing number attracted much attention after the relationship between the linking number of a closed ribbon and the writhing number of its axis, expressed by the White formula, was discovered independently by Călugăreanu [9], Fuller [21], Pohl

\footnotetext{
* Research by all three authors was partially supported by NSF under Grants CCR-00-86013 and EIA9972879. Work by the first author has also been supported by NSF Grants EIA-98-70724, EIA-01-31905, and CCR-97-32787, and by a grant from the U.S.-Israel Binational Science Foundation. Research by the second author was also partially supported by NSF under Grant CCR-97-12088.
} 


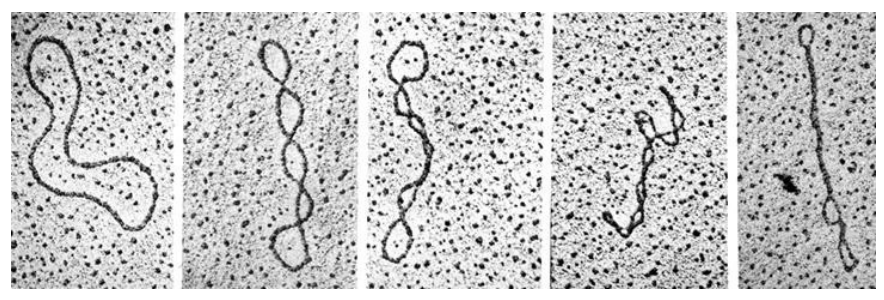

Fig. 1. Circular DNA takes on different supercoiling conformations in solution.

[27], and White [33]:

$$
L k=T w+W r
$$

Here the linking number, $L k$, is half the signed number of crossings between the two boundary curves of the ribbon, and the twisting number, $T w$, is half the average signed number of local crossing between the two curves. The non-local crossings between the two curves correspond to crossings of the ribbon axis, which are counted by the writhing number, $W r$. A small subset of the mathematical literature on the subject can be found in [3] and [20]. Besides the mathematical interest, the White Formula and the writhing number have received attention both in physics and in biochemistry [17], [23], [26], [30]. For example, they are relevant in understanding various geometric conformations we find for circular DNA in solution, as illustrated in Fig. 1 taken from [7]. By representing DNA as a ribbon, the writhing number of its axis measures the amount of supercoiling, which characterizes some of the DNA's chemical and biological properties [5].

This paper studies algorithms for computing the writhing number of a polygonal knot. Section 2 introduces background work and states our results. Section 3 relates the writhing number of a knot with the winding number of its Gauss map. Section 4 shows how to compute the writhing number in time less than quadratic in the number of edges of the knot. Section 5 discusses a simpler sweep-line algorithm and presents initial experimental results. Section 6 concludes the paper.

\section{Prior and New Work}

In this section we formally define the writhing number of a knot and review prior algorithms used to compute or approximate that number. We conclude by presenting our results.

Definitions. A knot is a continuous injection $K: \mathbb{S}^{1} \rightarrow \mathbb{R}^{3}$ or, equivalently, an oriented closed curve embedded in $\mathbb{R}^{3}$. We use the two-dimensional sphere of directions, $\mathbb{S}^{2}$, to represent the family of parallel projections in $\mathbb{R}^{3}$. Given a knot $K$ and a direction $z \in \mathbb{S}^{2}$, the projection of $K$ is an oriented, possibly self-intersecting, closed curve in a plane normal to $z$. We assume $z$ to be generic, that is, each crossing of $K$ in the direction $z$ is simple and identifies two oriented intervals along $K$, of which the one closer to the viewer is the overpass and the other is the underpass. We count the crossing as +1 if we 

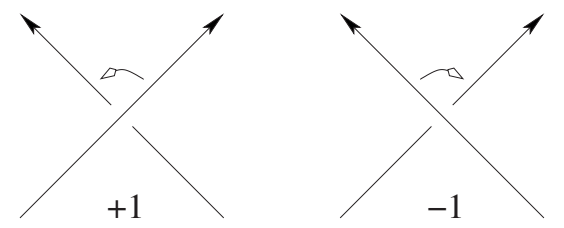

Fig. 2. The two types of crossings when two oriented intervals intersect.

can align the two orientations by rotating the overpass in counterclockwise order by an angle between 0 and $\pi$. Similarly, we count the crossing as -1 if the necessary rotation is in clockwise order. Both cases are illustrated in Fig. 2. The Tait or directional writhing number of $K$ in the direction $z$, denoted as $\operatorname{Dw}(z)$, is the sum of crossings counted as +1 or -1 as explained. The writhing number is the averaged directional writhing number, taken over all directions $z \in \mathbb{S}^{2}$,

$$
W r=\frac{1}{4 \pi} \int_{\mathbb{S}^{2}} D w(z) \mathrm{d} z .
$$

We note that a crossing in the projection along $z$ also exists in the opposite direction, along $-z$, and that it has the same sign. Hence $D w(z)=D w(-z)$, which implies that the writhing number can be obtained by averaging the directional writhing number over all points of the projective plane or, equivalently, over all antipodal points pairs $\{z,-z\}$ of the sphere.

Computing the Writhing Number. Several approaches to computing the writhing number of a smooth knot exactly or approximately have been developed. Consider an arclength parameterization $K: \mathbb{S}^{1} \rightarrow \mathbb{R}^{3}$, and use $K_{t}$ and $T_{t}$ to denote the position and the unit tangent vectors for $t \in \mathbb{S}^{1}$. The following double integral formula for the writhing number can be found in [27] and [31]:

$$
W r=\frac{1}{4 \pi} \int_{\mathbb{S}^{1}} \int_{\mathbb{S}^{1}} \frac{\left\langle T_{t} \times T_{s}, K_{t}-K_{s}\right\rangle}{\left\|K_{t}-K_{s}\right\|^{3}} \mathrm{~d} t \mathrm{~d} s .
$$

If the smooth knot is approximated by a polygonal knot, we can turn the right-hand side of (3) into a double sum and approximate the writhing number of the smooth knot [6], [26]. This can also be done in a way so that the double sum gives the exact writhing number of the polygonal knot [4], [24], [32].

Alternatively, we may base the computation of the writhing number on the directional version of the White formula, $L k=D w(z)+T w(z)$ for $z \in \mathbb{S}^{2}$. Recall that both the linking number and the twisting number are defined over the two boundary curves of a closed ribbon. Similar to the definition of $D w(z)$, the directional twisting number, $T w(z)$, is defined as half the sum of crossings between the two curves, each counted as +1 or -1 as described in Fig. 2 . We get (1) by integrating over $\mathbb{S}^{2}$ and noting that the linking number does not depend on the direction. This implies

$$
\begin{aligned}
W r & =L k-T w \\
& =D w(z)+T w(z)-\frac{1}{4 \pi} \int_{\mathbb{S}^{2}} T w(x) \mathrm{d} x .
\end{aligned}
$$


To compute the directional and the (average directional) twisting numbers, we expand $K$ to a ribbon, which amounts to constructing a second knot that runs alongside but is disjoint from $K$. Expressions for these numbers that depend on how we construct this second knot can be found in [24]. Le Bret [25] suggests fixing a direction $z$ and defining the second knot such that in the projection it always runs to the left of $K$. In this case we have $T w(z)=0$ and the writhing number is the directional writhing number for $z$ minus the twisting number.

A third approach to computing the writhing number is based on a result by Cimasoni [16], which states that the writhing number is the directional writhing number for a fixed direction $z$, plus the average deviation of the other directional writhing numbers from $D w(z)$. By observing that $D w(x)$ is the same for all directions $x$ in a cell $C$ of the decomposition of $\mathbb{S}^{2}$ formed by the Gauss maps $T$ and $-T$ (also referred to as the tangent indicatrix or tantrix in the literature [15], [29]), we get

$$
W r=D w(z)+\frac{1}{4 \pi} \sum_{C}\left[D w_{C}-D w(z)\right] A_{C},
$$

where $D w_{C}$ is $D w(x)$ for any one point $x$ in the interior of $C$, and $A_{C}$ is the area of $C$.

If applied to a polygonal knot, all three algorithms take time that is at least proportional to the square of the number of edges in the worst case.

Our Results. We present two new results. The first result can be viewed as a variation of (4) and a stronger version of (5). For a direction $x \in \mathbb{S}^{2}$ not on $T$ and not on $-T$, let $w(x)$ be its winding number with respect to $T$ and $-T$. As explained in Section 3, this means that $T$ and $-T$ wind $w(x)$ times around $x$.

Theorem A. For a knot $K$ and a direction $z$, we have

$$
W r=D w(z)-w(z)+\frac{1}{4 \pi} \int_{\mathbb{S}^{2}} w(x) \mathrm{d} x .
$$

Observe the similarity of this formula with (4), which suggests that the winding number can be interpreted as the directional twisting number for a ribbon, one of whose two boundary curves is $K$. We prove Theorem A in Section 3. We also extend the relation in Theorem A to open knots and give an algorithm that computes the average winding number in time proportional to the number of edges. Our second result is an algorithm that computes the directional writhing number for a polygonal knot in time sub-quadratic in the number of edges.

Theorem B. Given a polygonal knot $K$ with $n$ edges and a direction $z \in \mathbb{S}^{2}, D w(z)$ can be computed in time $O\left(n^{1.6+\varepsilon}\right)$, where $\varepsilon$ is an arbitrarily small positive constant.

Theorems A and B imply that the writhing number for a polygonal knot can be computed in time $\mathrm{O}\left(n^{1.6+\varepsilon}\right)$. As shown in Fig. 3, the number of crossings in a projection can be as large as quadratic in $n$. The sub-quadratic running time is achieved because the algorithm avoids checking each crossing explicitly. We also present a simpler sweepline algorithm that checks each crossing individually and therefore does not achieve the 


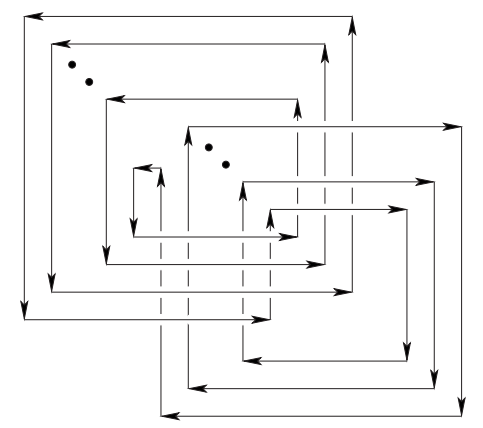

Fig. 3. A knot whose directional writhing number is quadratic in the number of edges.

worst-case running time of the algorithm in Theorem B. It is, however, fast when there are few crossings.

\section{Writhing and Winding}

In this section we develop our geometric understanding of the relationship between the writhing number of a knot and the winding number of its Gauss map. We define the Gauss map as the curve of critical directions, prove Theorem A, and give a fast algorithm for computing the average winding number.

Critical Directions. We specify a polygonal knot $K$ by the cyclic sequence of its vertices, $p_{0}, p_{1}, \ldots, p_{n-1}$ in $\mathbb{R}^{3}$. We use indices modulo $n$ and write $t_{i}=\left(p_{i+1}-\right.$ $\left.p_{i}\right) /\left\|p_{i+1}-p_{i}\right\|$ for the unit vector along the edge $p_{i} p_{i+1}$. Note that $t_{i}$ is also a direction in $\mathbb{R}^{3}$ and a point in $\mathbb{S}^{2}$. Any two consecutive points $t_{i}$ and $t_{i+1}$ determine a unique arc, which, by definition, is the shorter piece of the great circle that connects them. The cyclic sequence $t_{0}, t_{1}, \ldots, t_{n-1}$ thus defines an oriented closed curve $T$ in $\mathbb{S}^{2}$. We also need the antipodal curve, $-T$, which is the central reflection of $T$ through the origin.

The directions $y$ on $T$ and $-T$ are critical, in the sense that the directional writhing number changes when we pass through $y$ along a generic path in $\mathbb{S}^{2}$, and these are the only critical directions [16]. We sketch the proof of this claim for the polygonal case. It is clear that $y \in \mathbb{S}^{2}$ is critical only if it is parallel to a line that passes through a vertex $p_{i}$ and a point on an edge $p_{j} p_{j+1}$ of the knot that is not adjacent to $p_{i}$. There are $n(n-2)$ such vertex-edge pairs, each defining a great circle in $\mathbb{S}^{2}$. First, we note that only $n$ of these great circles actually carry critical points, namely, the great circles that correspond to $i=j-1$ and $i=j+2$. The reason for this is shown in Fig. 4, where we see that the writhing number does not change unless $p_{i}$ is separated from $p_{j} p_{j+1}$ by only one edge along the knot. Second, assuming $i=j-1$ we observe that the subset of directions along which $p_{i}$ projects onto $p_{i+1} p_{i+2}$ is the $\operatorname{arc} t_{i} u_{i}$ from $t_{i}$ to the direction $u_{i}=\left(p_{i+2}-p_{i}\right) /\left\|p_{i+2}-p_{i}\right\|$ in $\mathbb{S}^{2}$, and symmetrically the arc $-\left(t_{i} u_{i}\right)$ from $-t_{i}$ to $-u_{i}$. The subset of directions along which $p_{i+2}$ projects onto $p_{i} p_{i+1}$ are the arcs $u_{i} t_{i+1}$ and $-\left(u_{i} t_{i+1}\right)$. The points $t_{i}, u_{i}$, and $t_{i+1}$ lie on a common great circle and $u_{i}$ lies on the arc 

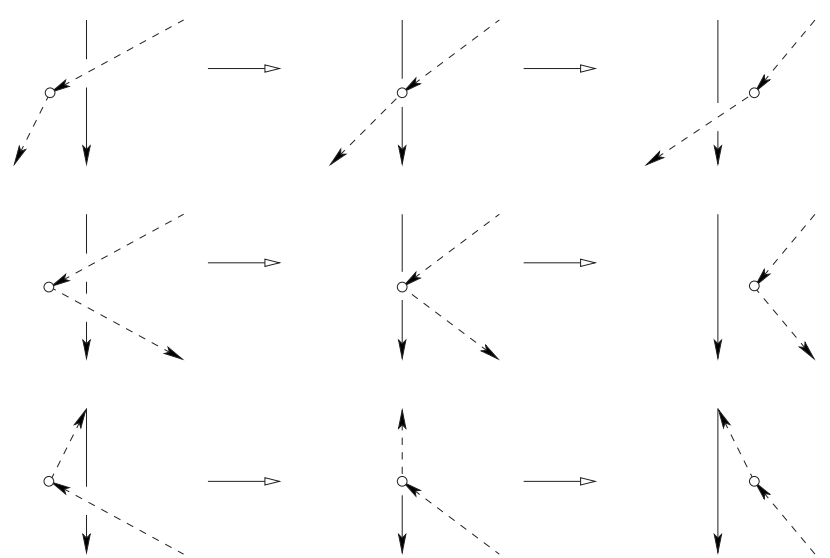

Fig. 4. In all three cases the viewing direction slides from left to right over the oriented great circle of directions defined by the hollow vertex and the solid edge. The directional writhing number changes only in the third case, where we lose a positive crossing.

$t_{i} t_{i+1}$. This implies that the concatenation of $t_{i} u_{i}$ and $u_{i} t_{i+1}$ is the arc $t_{i} t_{i+1}$, and that of $-\left(t_{i} u_{i}\right)$ and $-\left(u_{i} t_{i+1}\right)$ is the arc $-\left(t_{i} t_{i+1}\right)$. It follows that $T$ and $-T$ indeed comprise all critical directions.

Decomposition. The curves $T$ and $-T$ are both oriented, which is essential. We say a direction $x \in \mathbb{S}^{2}$ lies to the left of an oriented arc $u v$ if it lies in the open hemisphere to the left of the oriented great circle that contains $u v$. Equivalently, $x$ sees that great circle oriented in counterclockwise order. If $x$ passes from the left of an $\operatorname{arc} u v$ of $T$ to its right, then we either lose a positive crossing (as in the third row of Fig. 4), or we pick up a negative crossing. Either way the directional writhing number decreases by one. This motion corresponds to $-x$ passing from the right of the arc $-(u v)$ of $-T$ to its left. Since the directional writhing numbers at $x$ and $-x$ are the same, we decrease the directional writhing number by one in the opposite view as well. In other words, if $x$ moves from the left of an arc of $-T$ to its right, then the effect on the directional writhing number is the opposite from what it is for an arc of $T$. These simple rules allow us to keep track of the directional writhing number while moving around in $\mathbb{S}^{2}$. The curves $T$ and $-T$ decompose $\mathbb{S}^{2}$ into cells within which the directional writhing number is invariant. We can thus rewrite (2) as

$$
W r=\frac{1}{4 \pi} \sum_{C} D w_{C} A_{C}
$$

where the sum ranges over all cells $C$ of the decomposition, and $D w_{C}$ is the directional writhing number of any one point in the interior of $C$. Equation (5) of [16] can now be obtained by subtracting $D w(z)$ from $D w_{C}$ inside the sum and adding it outside the sum. This reformulation provides an algorithm for computing the writhing number:

Step 1. Compute $\operatorname{Dw}(z)$ for an arbitrary but fixed direction $z$.

Step 2. Construct the decomposition of $\mathbb{S}^{2}$ into cells, label each cell $C$ with $D w_{C}-$ $D w(z)$, and form the sum as in (5). 


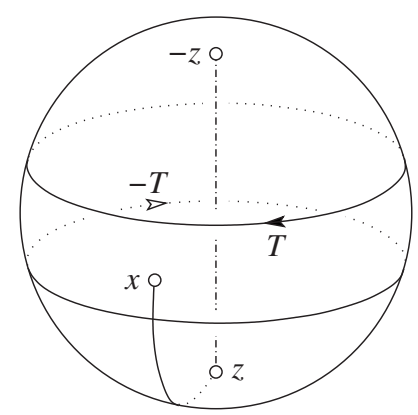

Fig. 5. The winding number counts the number of times $T$ separates $x$ from $-z$ and $-T$ separates $x$ from $z$.

The running time for Step 2 is $\Omega\left(n^{2}\right)$ in the worst case as there can be quadratically many cells. We improve the running time to $\mathrm{O}(n)$ and, at the same time, simplify the algorithm. First we prove Theorem A.

Winding Numbers. We now introduce a function $w$ over $\mathbb{S}^{2}$ that may be different from $D w$ but changes in the same way. In other words, $w(x)-w(z)=D w(x)-D w(z)$ for all $x, z \in \mathbb{S}^{2}$. This function is the winding number of a point $x \in \mathbb{S}^{2}$ with respect to the two curves $T$ and $-T$ that do not contain $x$. Observe that the space obtained by removing two points from the two-dimensional sphere is topologically an annulus. We fix non-critical, antipodal directions $z$ and $-z$ and define $w(x)$ equal to the number of times $T$ winds around the annulus obtained by removing $x$ and $-z$ plus the number of times $-T$ winds around the annulus obtained by removing $x$ and $z$. This is illustrated in Fig. 5, where $w(z)=w(-z)=1$ and $w(x)=2$. Here we count the winding of $T$ in counterclockwise order as seen from $x$ positive, and winding in clockwise order negative. Symmetrically, we count the winding of $-T$ in clockwise order as seen from $x$ positive, and winding in counterclockwise order negative. Imagine moving a point $y$ along $T$ and connecting $x$ to $y$ with a circular arc. Specifically, we use the circle that passes through $x, y$, and $-z$ and the arc with endpoints $x$ and $y$ that avoids $-z$. Symmetrically, we move $-y$ along $-T$ and connect $x$ to $-y$ with the appropriate arc of the circle passing through $x,-y$, and $z$. Locally at $x$ we observe continuous movements of the two arcs. Clockwise and counterclockwise movements cancel, and $w(x)$ is the number of times the first arc rotates in counterclockwise order around $x$ plus the number of times the second arc rotates in clockwise order around $x$. The winding number of $x$ is always an integer but can be negative.

Observe that $w$ indeed changes in the same way as $D w$ does. Specifically, $w$ drops by one if $x$ crosses $T$ from left to right, and it increases by one if $x$ crosses $-T$ from left to right. Starting from definition (2) of the writhing number, we thus get

$$
\begin{aligned}
W r & =\frac{1}{4 \pi} \int_{\mathbb{S}^{2}} D w(x) \mathrm{d} x \\
& =D w(z)+\frac{1}{4 \pi} \int_{\mathbb{S}^{2}}[D w(x)-D w(z)] \mathrm{d} x
\end{aligned}
$$




$$
\begin{aligned}
& =D w(z)+\frac{1}{4 \pi} \int_{\mathbb{S}^{2}}[w(x)-w(z)] \mathrm{d} x \\
& =D w(z)-w(z)+\frac{1}{4 \pi} \int_{\mathbb{S}^{2}} w(x) \mathrm{d} x,
\end{aligned}
$$

which completes the proof of Theorem A.

Signed Area Modulo 2. Observe that the writhing number changes continuously under deformations of the knot, as long as $K$ does not pass through itself. When $K$ performs a small motion during which it passes through itself there is a \pm 2 jump in $D w(z)$, while the average winding number changes only slightly. We use these observations to give a new proof of Fuller's relation [2], [22],

$$
1+W r=A_{T} / 2 \pi \quad(\bmod 2)
$$

where $A_{T}=\frac{1}{2} \int w(x) \mathrm{d} x$ is the signed area of the curve $T$ in $\mathbb{S}^{2}$. Note first that $1+W r=$ $A_{T} / 2 \pi \quad(\bmod 1)$ because both $D w(z)$ and $w(z)$ are integers. We start with $K$ being a circle in $\mathbb{R}^{3}$, in which case (6) holds because $W r=0$ and $A_{T}= \pm 2 \pi$. Other than continuous changes, we observe jumps of \pm 2 in $W r$ when $K$ passes through itself. Theorem A together with the fact that the fractional parts of $1+W r$ and $A_{T} / 2 \pi$ are the same implies that (6) is maintained during the deformation. Fuller's relation follows because every knot can be obtained from the circle by continuous deformation.

Computing the Average Winding Number. Three generic points $a, b, c \in \mathbb{S}^{2}$ define three arcs, which bound the spherical triangle $a b c$. Recall that the area of $a b c$ is the sum of angles minus $\pi$. We define the signed area of $a b c$ as $A=\alpha+\beta+\gamma-\pi$ if $a$ lies to the left of the oriented arc $b c$, and as $A=-\alpha-\beta-\gamma+\pi$ if it lies to the right. Let $z \in \mathbb{S}^{2}$ be a non-critical direction. As shown in Fig. 6, every arc $t_{i} t_{i+1}$ forms a unique spherical triangle $z t_{i} t_{i+1}$. Let $A_{i}$ be its signed area. The corresponding arc $-\left(t_{i} t_{i+1}\right)$ of $-T$ forms the antipodal spherical triangle $-\left(z t_{i} t_{i+1}\right)$ with signed area $-A_{i}$. The winding number of a direction $x \neq z$ can be obtained by counting the number of spherical triangles that contain it. To be more specific, we call a spherical triangle positive if its signed area is positive and negative if its signed area is negative. Let $P_{T}(x)$ and $N_{T}(x)$ be the numbers of positive and negative spherical triangles $z t_{i} t_{i+1}$ that contain $x$, and similarly let $P_{-T}(x)$ and $N_{-T}(x)$ be the numbers of positive and negative spherical triangles $-\left(z t_{i} t_{i+1}\right)$ that

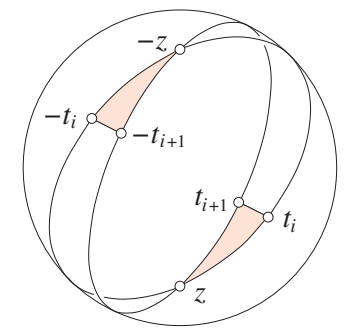

Fig. 6. The two spherical triangles defined by an arc of $T$ and its antipodal $\operatorname{arc}$ of $-T$. 
contain $x$. Then

$$
w(x)=\left[P_{T}(x)-N_{T}(x)\right]-\left[P_{-T}(x)-N_{-T}(x)\right] .
$$

To see this note that the equation is correct for a point $x$ near $z$ and remains correct as $x$ moves around and crosses arcs of $T$ and of $-T$. The average winding number is thus

$$
\begin{aligned}
\frac{1}{4 \pi} \int_{\mathbb{S}^{2}} w(x) \mathrm{d} x & =\frac{1}{4 \pi} \sum_{i=0}^{n-1} A_{i}-\frac{1}{4 \pi} \sum_{i=0}^{n-1}\left(-A_{i}\right) \\
& =\frac{1}{2 \pi} \sum_{i=0}^{n-1} A_{i} .
\end{aligned}
$$

Computing the sum in this equation is straightforward and takes only time $\mathrm{O}(n)$.

Open Knots. We define an open knot as a continuous injection $J:[0,1] \rightarrow \mathbb{R}^{3}$. Equivalently, it is an oriented curve, embedded in $\mathbb{R}^{3}$, with endpoints. The directional writhing number of $J$ is well defined, and the writhing number is the directional writhing number averaged over all parallel projections, as before. Assume $J$ is a polygonal curve specified by the sequence of its vertices, $p_{0}, p_{1}, \ldots, p_{n-1}$, and let $K$ be the knot obtained by adding the edge $p_{n-1} p_{0}$. The critical directions of $J$ differ in two ways from those of $K$ :

(i) there are critical directions of $K$ that are not critical for $J$, namely the ones whose definition includes a point of $p_{0} p_{n-1}$;

(ii) there are new critical directions, namely those defined by an endpoint ( $p_{0}$ or $\left.p_{n-1}\right)$ and another point of the polygon but not on the two adjacent edges.

To see that the directions in (ii) are indeed critical for $J$, examine the first two rows of Fig. 4. The hollow vertex is now an endpoint of $J$, so we remove one of the two dashed edges. Because of this change, the directional writhing number changes at the moment the hollow vertex passes over the solid edge. Changing the critical curve $T$ of $K$ to the critical curve $S$ of $J$ can thus be achieved by removing the arcs of Case (i) and adding the arcs of Case (ii). We illustrate this process in Fig. 7. To describe the process we define $v_{i}=\left(p_{i}-p_{0}\right) /\left\|p_{i}-p_{0}\right\|$, for $1 \leq i \leq n-1$, and $w_{j}=\left(p_{n-1}-p_{j}\right) /\left\|p_{n-1}-p_{j}\right\|$, for $0 \leq j \leq n-2$. Observe that $v_{2}=u_{0}, v_{n-2}=-u_{n-2}, v_{n-1}=w_{0}=-t_{n-1}, w_{1}=-u_{n-1}$, and $w_{n-3}=u_{n-3}$. We get the critical curve $S$ from $T$ by

1. removing the partial arcs $u_{n-3} t_{n-2}$ and $t_{0} u_{0}$, and the arcs $t_{n-2} t_{n-1}$ and $t_{n-1} t_{0}$,

2. adding the new paths $u_{0}=v_{2}, v_{3}, \ldots, v_{n-1}=-t_{n-1}$ and $-t_{n-1}=w_{0}, w_{1}, \ldots$, $w_{n-3}=u_{n-3}$.

Note that Step 2 adds a piece of $-T$, namely $-\left(u_{n-2} t_{n-1}\right)$ and $-\left(t_{n-1} u_{n-1}\right)$, to the new critical curve $S$. Symmetrically, we get $-S$ from $-T$. Everything we said earlier about the winding number of the critical curve $T$ of $K$ applies equally well to the critical curve $S$ of $J$. Similarly, all algorithms described in the subsequent sections apply to knots as well as to open knots. 


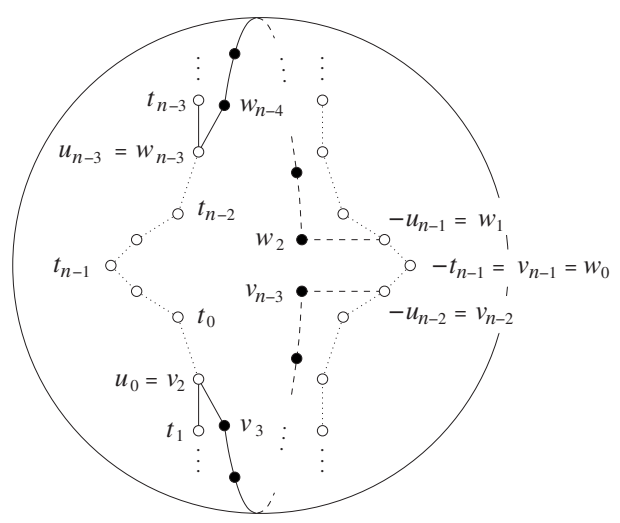

Fig. 7. The critical curves of the knot $K$ are marked by hollow vertices, and the additions required for the critical curves of the open knot $J$ are marked by solid black vertices.

\section{Computing Directional Writhing}

In this section we present an algorithm that computes the directional writhing number of a polygonal knot with $n$ edges in time roughly proportional to $n^{1.6}$. The algorithm uses complicated subroutines that may not lend themselves to an easy implementation.

Reduction to Five Dimensions. Assume without loss of generality that we view the knot $K$ from above, that is, in the direction of $z=(0,0,-1)$. Each edge $e_{i}=p_{i} p_{i+1}$ of $K$ is oriented. Another edge $e_{j}=p_{j} p_{j+1}$ that crosses $e_{i}$ in the projection either passes above or below and it either passes from left to right or from right to left. The four cases are illustrated in Fig. 8 and classified as positive and negative crossings according to Fig. 2. Letting $P_{i}$ and $N_{i}$ be the numbers of edges that form positive and negative crossings with $e_{i}$, the directional writhing number is

$$
D w(z)=\frac{1}{2}\left(\sum_{i=0}^{n-1} P_{i}-\sum_{i=0}^{n-1} N_{i}\right) .
$$

To compute the sums of the $P_{i}$ and $N_{i}$ efficiently, we map edges in $\mathbb{R}^{3}$ to points and half-spaces in $\mathbb{R}^{5}$. Specifically, let $\ell_{i}$ be the oriented line that contains the oriented edge $e_{i}$ and use Plücker coordinates as explained in [14] to map $\ell_{i}$ to a point $q_{i} \in \mathbb{R}^{5}$ or alternatively to a half-space $h_{i}$ in $\mathbb{R}^{5}$. The mapping has the property that $\ell_{i}$ and $\ell_{j}$ form a positive crossing if and only if $q_{i}$ lies in the interior of $h_{j}$. We use this correspondence

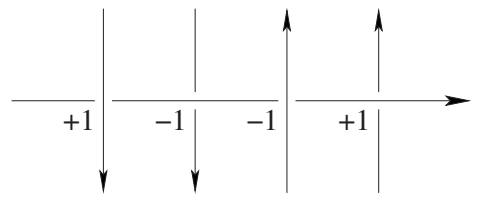

Fig. 8. The four ways an oriented edge can cross another. 
to compute $\sum_{i} P_{i}$ in two stages: first we collect the ordered pairs of oriented lines that form positive crossings, and second we count among them the pairs of edges that cross.

Recursive Algorithm. It is convenient to explain the algorithm in a slightly more general setting, where $X$ and $Y$ are sets of $x$ and $y$ oriented edges in $\mathbb{R}^{3}$. Let $P(X, Y)$ denote the number of pairs $(e, f) \in X \times Y$ that form positive crossings, and note that $\sum_{i} P_{i}=$ $P(X, Y)$ if $X$ is the set of edges of the knot $K$ and $Y=X$. We map $X$ to a set $Q$ of points and $Y$ to a set $H$ of half-spaces in $\mathbb{R}^{5}$. Let $r>0$ be a sufficiently large constant. A $1 / r$-cutting of $H$ and $Q$ is a collection of pairwise disjoint simplices covering $Q$ such that each simplex intersects at most $y / r$ hyperplanes bounding the half-spaces in $H$. We use the algorithm in [1] to compute a $1 / r$-cutting consisting of $s$ simplices in time $\mathrm{O}(x+y)$, where $s$ is at most $r^{4} \log r$ times a constant independent of $r$. For each simplex $\Delta_{k}$ in the cutting, define

$$
\begin{aligned}
X_{k} & =\left\{e_{i} \in X \mid q_{i} \in \Delta_{k}\right\}, \\
Y_{k} & =\left\{f_{j} \in Y \mid \text { bd } h_{j} \cap \Delta_{k} \neq \emptyset\right\}, \\
Z_{k} & =\left\{f_{j} \in Y \mid \Delta_{k} \subseteq h_{j}\right\} .
\end{aligned}
$$

Letting $x_{k}=\operatorname{card} X_{k}$ and $y_{k}=\operatorname{card} Y_{k}$, we have $\sum_{k} x_{k}=x$ and $y_{k} \leq y / r$. By construction, every $(e, f) \in X_{k} \times Z_{k}$ defines a pair of lines that form a positive crossing. For each simplex $\Delta_{k}$, we count the edge pairs $(e, f) \in X_{k} \times Z_{k}$ that form positive crossings, and let $P_{k}$ be the number of such pairs. Then

$$
P(X, Y)=\sum_{k=1}^{s}\left[P\left(X_{k}, Y_{k}\right)+P_{k}\right]
$$

Note that $P_{k}$ is the number of crossings between projections of the line segments in $X_{k}$ and in $Z_{k}$. We can therefore use the algorithm in [13] to compute all numbers $P_{k}$, for $1 \leq k \leq s$, in time $\mathrm{S}(x, y)=\mathrm{O}\left(x^{2 / 3} y^{2 / 3} \log x+x \log x+y \log y\right)$. We recurse to compute the $P\left(X_{k}, Y_{k}\right)$ and stop the recursion when $y \leq r$. The running time of this algorithm is at most

$$
\begin{aligned}
\mathrm{T}(x, y) & =\mathrm{S}(x, y)+\sum_{k=1}^{s} \mathrm{~T}\left(x_{k}, y / r\right) \\
& =\mathrm{O}\left(y^{4+\varepsilon}+x \log ^{2} x\right)
\end{aligned}
$$

for any $\varepsilon>0$, provided $r=r(\varepsilon)$ is sufficiently large.

Improving the Running Time. We improve the running time of the algorithm by taking advantage of the symmetry of the mapping to $\mathbb{R}^{5}$. Specifically, a point $q_{i}$ lies in the interior of a half-space $h_{j}$ if and only if the point $q_{j}$ lies in the interior of the halfspace $h_{i}$. We proceed as above, but switch the roles of points and half-spaces when $x^{4}$ becomes less than $y$. That is, if $x^{4}<y$, then we map the edges in $X$ to half-spaces and the edges in $Y$ to points. By our above analysis, the running time is then less than 
$\mathrm{T}(y, x)=\mathrm{O}\left(x^{4+\varepsilon}+y \log ^{2} y\right)=\mathrm{O}\left(y^{1+\varepsilon}\right)$. The overall running time is thus less than

$$
\begin{aligned}
& \mathrm{T}(x, y)=\left\{\begin{array}{lll}
\mathrm{S}(x, y)+\sum_{k=1}^{s} \mathrm{~T}\left(x_{k}, \frac{y}{r}\right) & \text { if } & x^{4} \geq y, \\
c y^{1+\varepsilon} & \text { if } & x^{4}<y
\end{array}\right. \\
& =\mathrm{O}\left((x y)^{0.8+\delta}+(x+y)^{1+\delta}\right) \text {, }
\end{aligned}
$$

where $c$ is a positive constant and $\delta$ is any real larger than $\varepsilon$. It follows that $\sum_{i} P_{i}$ can be computed in time $\mathrm{O}\left(n^{1.6+\varepsilon}\right)$, for any constant $\varepsilon>0$. Similarly, $\sum_{i} N_{i}$ and therefore the directional writhing number, $D w(z)$, can be computed within the same time bound, thereby proving Theorem B.

We remark that the technique described in this section can also be used to compute the linking number between two polygonal knots with $n$ and $m \leq n$ edges in time $\mathrm{O}\left(n^{1.6+\varepsilon}\right)$.

\section{Experiments}

In this section we sketch a sweep-line algorithm that computes the writhing number of a polygonal knot using Theorem $\mathrm{A}$. We implemented the algorithm in $\mathrm{C}++$ using the LEDA software library and compared it with two versions of the algorithm based on the double integral in (3). We did not implement any version of Le Bret's algorithm mentioned in Section 2 since it is based on a formula similar to Theorem A and can be expected to perform about the same as our sweep-line algorithm.

Sweep-Line Algorithm. Theorem A expresses the writhing number of a knot $K$ as the sum of three terms. Accordingly, we compute the writhing number in three steps:

Step 1. Compute the directional writhing number for an arbitrary but fixed noncritical direction $z, D w(z)$.

Step 2. Compute the winding number of $z$ relative to the Gauss maps $T$ and $-T$, $w(z)$.

Step 3. Compute the average winding number by summing the signed areas of the spherical triangles $z t_{i} t_{i+1},(1 / 2 \pi) \sum_{i} A_{i}$.

Return $D w(z)-w(z)+(1 / 2 \pi) \sum_{i} A_{i}$.

Instead of using the algorithm described in Section 4, we implemented Step 1 using a sweep-line algorithm [18], which reports the $m$ crossing pairs formed by the $n$ edges in time $\mathrm{O}((n+m) \log n)$. Steps 2 and 3 are both computed in a single traversal of the spherical polygons $T$ and $-T$, keeping track of the accumulated angle and the signed area as we go. The running time of the traversal is only $\mathrm{O}(n)$.

Double-Sum Algorithm. We compare the implementation of the sweep-line algorithm with two implementations of (3). Write $e_{i}=p_{i+1}-p_{i}$ for the unnormalized tangent vector. Following [6] and [26], we discretize (3) to

$$
W x=\frac{1}{4 \pi} \sum_{i=0}^{n-1} \sum_{j \neq i} \frac{\left\langle e_{j} \times e_{i}, p_{j}-p_{i}\right\rangle}{\left\|p_{j}-p_{i}\right\|^{3}} .
$$


We note that $W x$ is not the writhing number of the polygonal knot, but it converges to the writhing number of a smooth knot if the polygonal approximation is progressively refined to approach that knot [10].

Alternatively, we may discretize the double integral in such a way that the result is the writhing number of the approximating polygonal knot. Given two edges $e_{i}$ and $e_{j}$, we measure the area of the two antipodal quadrangles in $\mathbb{S}^{2}$ along whose directions we see the edges cross. The area of one of the quadrangles is the sum of angles minus one full angle, $\alpha+\beta+\gamma+\delta-2 \pi$. The absolute value of the signed area $A_{i j}$ is the same, and its sign depends on whether we see a positive or a negative crossing. We thus have

$$
W r=\frac{1}{4 \pi} \sum_{i=0}^{n-1} \sum_{j \neq i} A_{i j}
$$

Straightforward vector geometry and trigonometry can be used to derive analytical formulas for the $A_{i j}$ [4], [24].

Comparison. We compare the three implementations using a sequence of polygonal approximations of an artificially created smooth knot. It has the form of the infinity symbol, $\infty$, and is fairly flat in $\mathbb{R}^{3}$, with only a small gap in the middle. Because the knots are fairly flat, most of their parallel projections have one crossing and the writhing number is just a little smaller than 1.0. Figure 9 shows that the algorithms that compute the exact writhing numbers for polygonal approximations converge faster to the writhing number of the smooth knot than the algorithm implementing (7). Figure 10 shows how much faster the sweep-line algorithm is than both implementations of the double-sum algorithm. Let $n$ be the number of edges. The graphs suggest that the running time of the sweep-line algorithm is $\mathrm{O}(n)$ and the running times of the two implementations of the double-sum algorithm are $\Theta\left(n^{2}\right)$. We observe the linear bound whenever we approximate a smooth knot by a polygon, since for generic projections the number of crossings as well as the number of edges simultaneously intersected by the sweep-line are independent of the total number of edges.

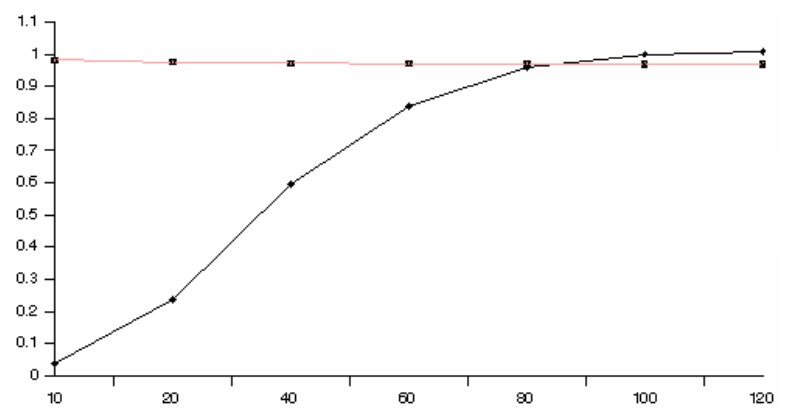

Fig. 9. Comparing convergence rates between $W r$ (upper curve) and $W x$ (lower curve). For each tested approximation of the $\infty$-knot, we draw the number of vertices along the horizontal axis and the writhing number along the vertical axis. 


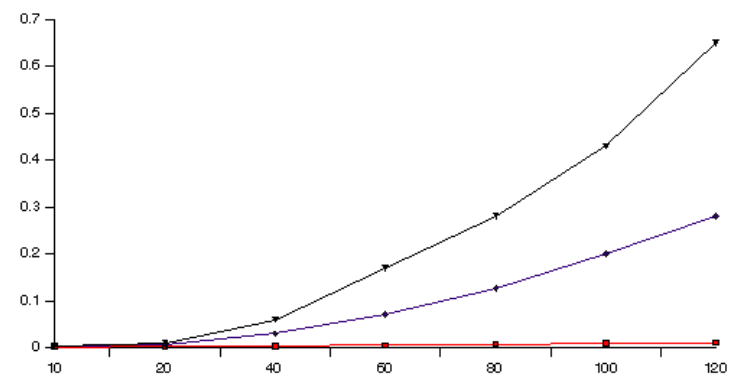

Fig. 10. Comparing the running times of the sweep-line algorithm (lower curve) and the two implementations of the double-sum algorithm: approximate (middle curve) and exact (upper curve). The $x$-axis and $y$-axis represent the number of vertices in the curve, and the running time of the algorithm respectively.

Protein Backbones. We present some preliminary experimental results obtained with the three implementations. All experiments are carried out on a SUN workstation, with a $333 \mathrm{MHz}$ UltraSPARC-IIi CPU, and $256 \mathrm{MB}$ memory. Short of conformation data of long DNA strands, we decided to run our algorithms on a modest collection of open knots representing protein backbones, down-loaded from the protein data bank [28]. We modified the algorithms to account for the missing edge in the data, as explained in Section 3. Figure 11 displays the four backbones chosen for our experimental study. Table 1 presents some of our findings.

Thick Knots. Even though the writhing number of a polygonal knot can be as large as quadratic in the number of edges, all four protein backbones in Fig. 11 have writhing numbers that are significantly smaller than the numbers of edges. If a knot is made out of rope with non-zero thickness, then the quadratic bound can be achieved only if the ratio of length over cross-section radius is sufficiently high. Specifically, the writhing number of a knot of length $L$ with an embedded tubular neighborhood of radius $R$ is less than $\frac{1}{4}(L / R)^{4 / 3}[11]$. Such "thick" knots can be used to capture the fact that the edges of a protein backbone are about as long as they are thick. A backbone with $n$ edges thus has a writhing number at most some constant times $n^{4 / 3}$. Examples which show that the upper bound is asymptotically tight can be found in [8], [12], and [19].

\section{Discussion}

In this paper we have described the relationship between the writhing number of a knot in $\mathbb{R}^{3}$ and the winding number of its Gauss map. Based on this relationship, we have given an algorithm that computes the writhing number of a polygonal knot in time less than quadratic in the number of edges. We implemented a different algorithm whose running time depends on the number of crossings in a projection and tested the software on open knots describing protein backbones. It would be interesting to expand these experiments to see whether there is a correlation between the writhing numbers and the common categorization of folding patterns into protein families. To approach this question, it might be necessary to consider knots on a range of scale levels and look at the writhing number as a function of scale. 

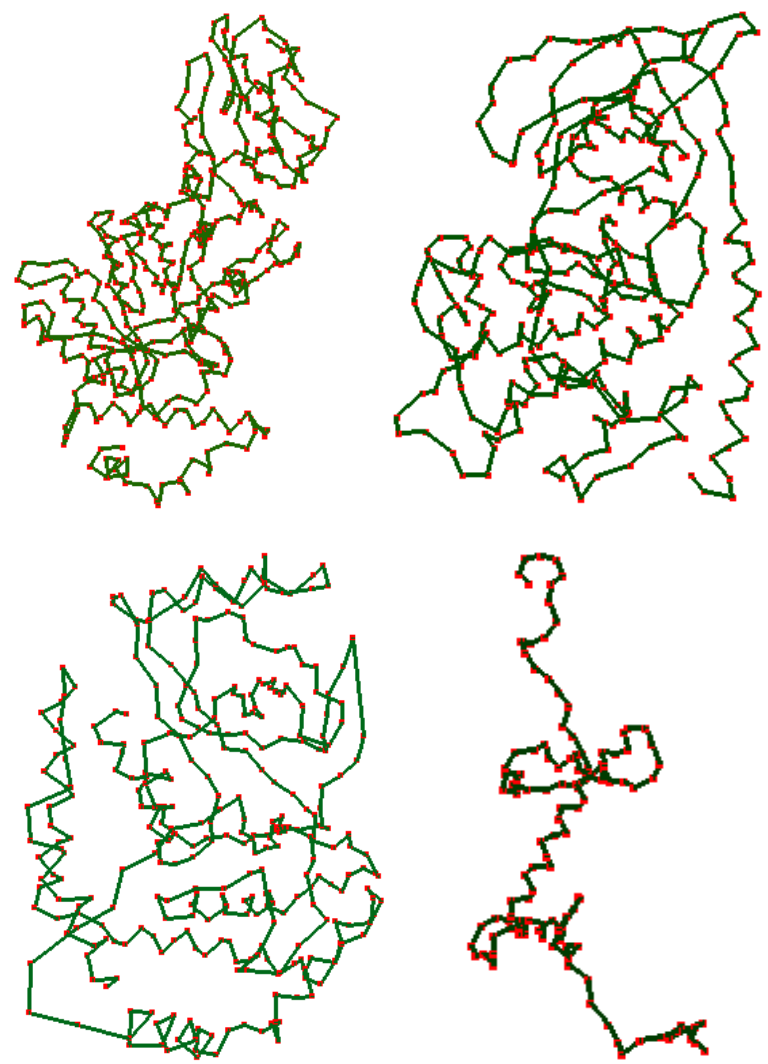

Fig. 11. The open knots modeling the backbone of the protein conformations stored in the PDB files 1AUS.pdb (upper left), 1CDK.pdb (upper right), 1CJA.pdb (lower left), and 1EQZ.pdb (lower right).

Table 1. Four protein backbones modeled by open polygonal knots.

\begin{tabular}{|l||c|c||c|c|c||r|r|}
\hline \multicolumn{1}{|c||}{} & \multicolumn{2}{c||}{ Size $^{*}$} & \multicolumn{3}{c||}{ Time } & \multicolumn{2}{c|}{ Writhing number } \\
\cline { 2 - 8 } Data & $n$ & $m$ & SwL & DS $_{\mathrm{a}}$ & $\mathrm{DS}_{\mathrm{e}}$ & $W r$ & $W x$ \\
\hline 1AUS & 439 & 122 & 0.09 & 3.93 & 9.28 & 22.70 & 17.87 \\
1CDK & 343 & 111 & 0.06 & 2.39 & 5.62 & 7.96 & 6.01 \\
1CJA & 327 & 150 & 0.06 & 2.19 & 5.10 & 12.14 & 10.43 \\
1EQZ & 125 & 18 & 0.02 & 0.31 & 0.73 & 4.78 & 3.37 \\
\hline
\end{tabular}

*The size of the problem is measure by the number of edges, $n$, and the number of crossings in the chosen projection, $m$. The time the sweep-line ( $\mathrm{SwL}$ ), the approximate double-sum $\left(\mathrm{DS}_{\mathrm{a}}\right)$, and the exact double-sum $\left(\mathrm{DS}_{\mathrm{e}}\right)$ algorithms take is measured in seconds. $W x$ is an approximation of the writhing number for polygonal data. 


\section{Acknowledgment}

We thank Michael Levitt for motivating us to look at the problem of computing writhing numbers and Sariel Har-Peled and Robert K.-Z. Tan for helpful discussions.

\section{Appendix}

Table 2 provides a list of notation used in this paper.

Table 2. Notation for important geometric concepts, functions, variables, and constants.

\begin{tabular}{ll}
\hline $\mathbb{R}^{3}$ & Three-dimensional Euclidean space \\
$\mathbb{S}^{2}$ & Sphere of directions \\
$x, z$ & Generic directions \\
$K: \mathbb{S}^{1} \rightarrow \mathbb{R}^{3}$ & (Closed) knot \\
$J:[0,1] \rightarrow \mathbb{R}^{3}$ & Open knot \\
$D w, W r$ & (Directional) writhing number \\
$W x$ & Approximation of $W r$ \\
$L k, T w, w$ & Linking, twisting, winding number \\
$n, m$ & \\
$p_{i}$ & Number of edges, crossings \\
$e_{i}=p_{i+1}-p_{i}$ & Vertices \\
$T,-T, S,-S$ & Directed edges \\
$t_{i}, u_{i}, v_{i}, w_{i}$ & Curves of critical directions \\
$A$ & Vertices of critical curves \\
& Area and signed area \\
$\ell_{i}$ & \\
$q_{i}, h_{i}$ & Oriented lines in $\mathbb{R}^{3}$ \\
$X, Y, x, y$ & Points, half-spaces in $\mathbb{R}^{5}$ \\
$\Delta k, r$ & Sets, numbers of oriented edges \\
$\mathrm{T}, \mathrm{S}$ & Simplices in, quality of cutting \\
$\varepsilon, \delta$ & Running time \\
\hline
\end{tabular}

\section{References}

1. P. K. Agarwal and J. Matoušek. On range searching with semialgebraic sets. Discrete Comput. Geom. 11 (1994), 393-418.

2. J. Aldinger, I. Klapper, and M. Tabor. Formulae for the calculation and estimation of writhe. J. Knot Theory Ramifications 4 (1995), 343-372.

3. A. M. Amilibia and J. J. N. Ballesteros. The self-linking number of a closed curve in $\mathbb{R}^{n}$. J. Knot Theory Ramifications 9 (2000), 491-503.

4. T. Banchoff. Self-linking numbers of space polygons. Indiana Univ. Math. J. 25 (1976), 1171-1188.

5. W. R. Bauer, F. H. C. Crick, and J. H. White. Supercoiled DNA. Scientific American 243 (1980), 118-133.

6. K. Brakke. Surface evolver software documentation. http://www.geom.umn.edu/software/evolver/.

7. D. Brutlag. DNA topology \& topoisomerases. http://cmgm.stanford.edu/biochem201/Handouts/DNAtopo.html, 2000

8. G. Buck. Four-thirds power law for knots and links. Nature. 392 (1998), 238-239. 
9. G. Călugăreanu. Sur les classes d'isotopie des noeuds tridimensionnels et leurs invariants. Czech. Math. J. 11 (1961), 588-625.

10. J. Cantarella. On comparing the writhe of a smooth curve to the writhe of an inscribed polygon. To appear in SIAM J. Numer. Anal.

11. J. Cantarella, D. DeTurck, and H. Gluck. Upper bounds for the writhing of knots and the helicity of vector fields. In Proc. Conf. in Honor of 70th Birthday of Joan Birman, J. Gilman, X. Lin, and W. Menasco, eds., 2000, pp. 1-22.

12. J. Cantarella, R. Kusner, and J. Sullivan. Tight knot values deviate from linear relation. Nature 392 (1998), 237-238.

13. B. Chazelle. Cutting hyperplanes for divide-and-conquer. Discrete Comput. Geom. 9 (1993), 145-158.

14. B. Chazelle, H. Edelsbrunner, L. J. Guibas, M. Sharir, and J. Stolfi. Lines in space: combinatorics and algorithms. Algorithmica 15 (1996), 428-447.

15. S. S. Chern. Curves and surfaces in Euclidean space. In Studies in Global Geometry and Analysis, S. S. Chern, ed. Mathmatical Association of America Washington, DC, 1967, pp. 16-56.

16. D. Cimasoni. Computing the writhe of a knot. J. Knot Theory Ramifications 10 (2001), 387-395.

17. F. H. C. Crick. Linking numbers and nucleosomes. Proc. Nat. Acad. Sci. U.S.A. 73 (1976), 2639-2643.

18. M. de Berg, M. van Kreveld, M. Overmars, and O. Schwarzkopf. Computational Geometry: Algorithms and Applications. Springer-Verlag, New York, 1997.

19. Y. Diao and C. Ernst. The complexity of lattice knots. Topology Appl. 90 (1998), 1-9.

20. M. H. Eggar. On White's formula. J. Knot Theory Ramifications 9 (2000), 611-615.

21. F. B. Fuller. The writhing number of a space curve. Proc. Nat. Acad. Sci. U.S.A. 68 (1971), 815-819.

22. F. B. Fuller. Decomposition of the linking number of a closed ribbon: a problem from molecular biology. Proc. Nat. Acad. Sci. U.S.A. 75 (1978), 3557-3561.

23. R. D. Kamien. Local writhing dynamics. European Phys. J. B 1 (1998), 1-4.

24. K. Klenin and J. Langowski. Computation of writhe in modeling of supercoiled DNA. Biopolymers $\mathbf{5 4}$ (2000), 307-317.

25. M. Le Bret. Catastrophic variation of twist and writhing of circular DNAs with constraint? Biopolymers 18 (1979), 1709-1725.

26. M. Levitt. Protein folding by restrained energy minimization and molecular dynamics. J. Mol. Biol. 170 (1983), 723-764.

27. W. F. Pohl. The self-linking number of a closed space curve. J. Math. Mech. 17 (1968), 975-985.

28. Protein Data Bank. http://www.rcsb.org/pdb/.

29. B. Solomon. Tantrices of spherical curves. Amer. Math. Monthly 103 (1996), 30-39.

30. D. Swigon, B. D. Coleman, and I. Tobias. The elastic rod model for DNA and its application to the tertiary structure of DNA minicircles in mononucleosomes. Biophys. J. 74 (1998), 2515-2530.

31. M. Tabor and I. Klapper. The dynamics of knots and curves (Part I). Nonlinear Sci. Today 4 (1994), 7-13.

32. A. V. Vologodskii, V. V. Anshelevich, A. V. Lukashin, and M. D. Frank-Kamenetskii. Statistical mechanics of supercoils and the torsional stiffness of the DNA double helix. Nature 280 (1979), 294-298.

33. J. White. Self-linking and the Gauss integral in higher dimensions. Amer. J. Math. XCI (1969), 693-728.

Received April 6, 2002, and in revised form January 27, 2003, and July 29, 2003.

Online publication April 19, 2004. 